\title{
Closed-Form BER Expressions of QPSK constellation for Uplink Non-Orthogonal Multiple Access (NOMA)
}

\author{
Xiaolu Wang, Student Member, IEEE, Fabrice Labeau, Senior Member, IEEE, and Lin Mei, Member, IEEE
}

\begin{abstract}
Non-orthogonal multiple access (NOMA) is an attractive multiple access technique to achieve the optimal system capacity region. A great deal of recent attention has been devoted to the study of the NOMA system capacity performance. However, the exact BER expressions of NOMA systems have not been derived yet. In this letter, we provide the exact closed-form BER expressions of the QPSK constellation for an uplink NOMA system over an additive white Gaussian noise (AWGN) channel. Finally, the validity of our derived BER expressions is verified through simulations.
\end{abstract}

Index Terms-5G, non-orthogonal multiple access (NOMA), bit error rate (BER), error propagation.

\section{INTRODUCTION}

$\mathbf{N}$ ON-ORTHOGONAL multiple access (NOMA), where multiple users are multiplexed in the power-domain $[1,2]$, has been considered as a promising multiple access technology for fifth-generation $(5 \mathrm{G})$ communications in both uplink [3-5] and downlink [3, 6, 7]. In principle, NOMA works by exploiting the relative disparities in channel quality from different users, while using the entire bandwith for each user. Due to its potential, NOMA has been studied intensely in the last few years, in terms of power allocation [3], outage performance [6], and user pairing [7].

In these contributions, many problems are addressed from the perspective of the channel capacity/system capacity based on the Shannon formula [1-3, 6, 7]. However, these researches assume that there is no error propagation when implementing the successive interference cancellation (SIC) process at the receiver, which is not consistent with actual wireless communications. Besides, there are few accurate theoretical bit error rate (BER) expressions of the NOMA scheme provided in the literature, though system performance simulation results are shown under various environments in the literature [5]. In [8], the BER performance in an asynchronous uplink NOMA scheme is investigated. It is assumed that the relative time

This work was supported by Hydro-Quebec, the Natural Sciences and Engineering Research Council of Canada and McGill University in the framework of the NSERC/Hydro-Quebec/McGill Industrial Research Chair in Interactive Information Infrastructure for the Power Grid (IRCPJ 406021-14), the National Natural Science Foundation Program of China (No.61671179), the National Basic Research Program of China (2013CB329003), Science and Technology on Information Transmission and Dissemination in Communication Networks Laboratory (EX156410046) and China Scholarship Council.

Xiaolu Wang is with the Communication Research Center, Harbin Institute of Technology, Harbin 150001, China, and is also with the Department of Electrical and Computer Engineering, McGill University, Montreal, QC H3A 0E9, Canada (e-mail: xiaolu_wang@outlook.com; xiaolu.wang@mail.mcgill.ca).

Fabrice Labeau is with the Department of Electrical and Computer Engineering, McGill University, Montreal, QC H3A 0E9, Canada (e-mail: fabrice.labeau@mcgill.ca).

Lin Mei is with the Communication Research Center, Harbin Institute of Technology, Harbin 150001, China (e-mail: meilin@hit.edu.cn). offset between users follows uniform distribution in the asynchronous NOMA scheme, and the total error propagation can approximately follow the Gaussian distribution. As a result, the approximated asynchronous uplink NOMA BER expressions are obtained. In this letter, the uplink NOMA model is assumed to be perfectly synchronized, so the error propagation does not follow the Gaussian distribution. Therefore, it is necessary to investigate the effects of error propagation on the BER performance in a synchronous uplink NOMA scheme. In consideration of the foregoing, the error propagation is taken into account to analyze the BERs of users in a synchronous uplink NOMA scheme in this letter, where each user adopts unrotated Gray code mapping QPSK. The exact closed-form expressions of BER for QPSK synchronous uplink NOMA under an additive white Gaussian noise (AWGN) channel are presented, for the first time in the literature, to the best of our knowledge.

The remainder of this letter is organized as follows. Section II briefly describes the system model of the uplink NOMA. In Section III, the explicit closed-form BER expressions of QPSK constellation for the uplink NOMA system are presented. Simulation results are provided to verify the derived closedform BER expressions in Section IV. Finally, conclusions are drawn in Section V.

\section{SYSTEM MODEL}

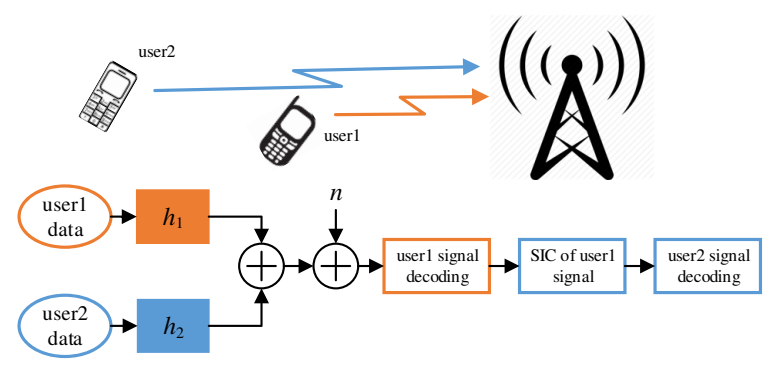

Fig. 1. Two user uplink NOMA scheme applying with SIC receiver

This letter considers the uplink of a NOMA system which consists of one base station (BS) and two users. The corresponding schematic of the uplink NOMA channel is illustrated in Fig. 1. The two users share the same time-frequency resources to transmit signals. The terminals of two users have individual power constraints, and the power control to users' uplink transmissions is assumed as given system parameters. As this letter focuses on the analysis of BER performance, the power control mechanism is not considered here. The received signal at the BS is represented as

$$
r=h_{1} t_{1}+h_{2} t_{2}+n
$$


where $h_{1}$ and $h_{2}$ are the channel gains between user1 and user2, respectively, and the BS, which consist of path-loss, shadow fading, and small scale fading. The small scale fading is assumed to be constant within the block duration time for simplicity. $t_{1}$ and $t_{2}$ are the corresponding transmitted signals of the two users with power $P_{1}$ and $P_{2}$ respectively. $n$ is the zero-mean AWGN with average power $P_{n}$. For simplicity, we assume perfect carrier recovery, symbol synchronization and perfect channel knowledge at the BS. Single-carrier frequency division multiple access (SC-FDMA) has been adopted in the uplink of the 4th generation (4G) mobile communication system [9]. Therefore, it is assumed that the two users adopt the single-carrier frequency division multiplexing (SC-FDM) in the uplink NOMA scheme [4] and $\left|h_{1}\right|>\left|h_{2}\right|$.

At the BS, the SIC process is implemented to decode the two users' signals. The optimal order of decoding is in the order of decreasing channel gain, and it is assumed that $P_{1}\left|h_{1}\right|^{2}>P_{2}\left|h_{2}\right|^{2}$. Therefore, the BS first decodes user1's signals, treating the received user2's signals as interuser interference (IUI). Then the BS subtracts the user1's reconstructed signals from the aggregate received signals $r$. If the BS correctly decodes user1 signals, user2 signals can be decoded without interference from user1. Otherwise, there will be error propagation from user1 when decoding user2 signals. Here, the signal to noise ratio (SNR) of the two users are defined as $\mathrm{SNR}_{1}=P_{1}\left|h_{1}\right|^{2} / P_{n}$ and $\mathrm{SNR}_{2}=P_{2}\left|h_{2}\right|^{2} / P_{n}$.

\section{ClOSED-FORM EXPRESSIONS FOR THE EXACT BER OF QPSK CONSTELLATION}

In this section, we present the explicit closed-form expressions of the QPSK BER for the two users. Assume that if without AWGN, $2 d(d>0)$ is the minimum distance between the received constellation points of user1 [10]. Hence $2 d / \sqrt{A}$ is the minimum distance between the received constellation points of user2, where $A=\mathrm{SNR}_{1} / \mathrm{SNR}_{2}$.

Fig. 2 shows user1 QPSK signal constellations in the presence of user2 QPSK signal constellations as IUI, where each constellation point is represented by four bits $\left(i_{1} i_{2}, i_{3} i_{4}\right)$. $\left(i_{1} i_{2}\right)$ are the two bits of user1 QPSK and $\left(i_{3} i_{4}\right)$ are the two bits of user2 QPSK. All the symbols are assumed to be transmitted equally likely. Due to the existence of IUI from user2, each received user1 original constellation point is transferred to four possible constellation points, e.g., the user1 original constellation point (10) is transferred to four possible constellation points $(10,10),(10,00),(10,11),(10,01)$.

For user1 QPSK 1st bit $i_{1}$, there are four possible cases (see four dashed ellipses) in which the bit $i_{1}$ is decoded incorrectly. When $(10,10)$ is sent, zero is the decision boundary and the constellation point is at a distance $(d+d / \sqrt{A})$ from the decision boundary. If the AWGN exceeds $(d+d / \sqrt{A})$, a bit error will occur. Therefore, considering these four cases, the probability that the first bit $i_{1}$ is in error is

$$
\mathcal{P}_{i_{1}}=\frac{1}{4}\left[\operatorname{Pr}\left(d-\frac{d}{\sqrt{A}}<|n|\right)+\operatorname{Pr}\left(d+\frac{d}{\sqrt{A}}<|n|\right)\right],
$$

where $\operatorname{Pr}(\cdot)$ represents the probability. The average BER of QPSK scheme can be obtained by averaging the 2 bits $\left(i_{1} i_{2}\right)$ error probability [11]. $\mathcal{P}_{i_{1}}$ equals $\mathcal{P}_{i_{2}}$, so the closed-form BER expression of user1 can be formulated as

$$
\mathcal{P}_{1}=\frac{1}{2}\left[Q\left(\frac{d-d / \sqrt{A}}{\sqrt{P_{n} / 2}}\right)+Q\left(\frac{d+d / \sqrt{A}}{\sqrt{P_{n} / 2}}\right)\right],
$$

where $Q(\cdot)$ denotes $Q$ function which is defined as $Q(x)=$ $1 / \sqrt{2 \pi} \int_{x}^{\infty} \exp \left(-u^{2} / 2\right) d u$.

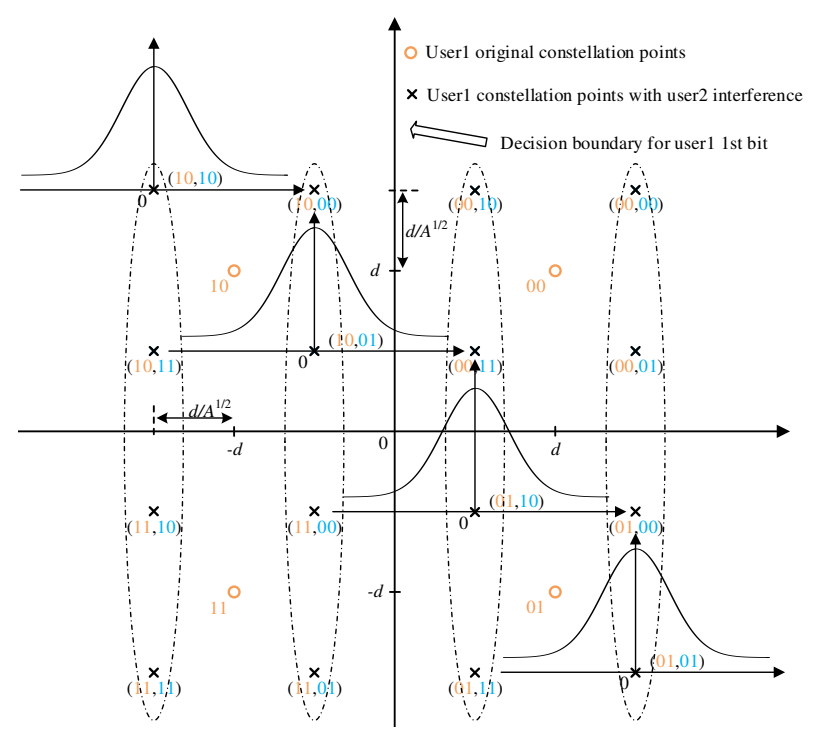

Fig. 2. Signal-space diagram for the user1 QPSK 1st bit (orange color represents the user1 QPSK bits, blue color the user2 QPSK bits.).

Whether the decoding result of bit $i_{1}$ is correct effects decoding the user2 $1 \mathrm{st}$ bit $i_{3}$. However, the bit $i_{2}$ has no influence on the decoding of bit $i_{3}$. For simplicity, it is assumed that the decoding of bit $i_{2}$ is correct when decoding bit $i_{3}$. Therefore, there are two classes (class I: correct decoding bit $i_{1}$ and class II: wrong decoding bit $i_{1}$ ) after SIC for decoding user2 1 st bit $i_{3}$.

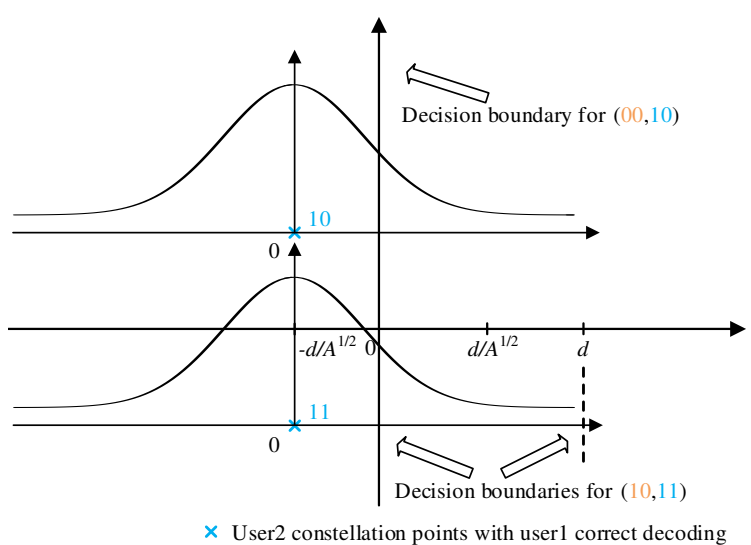

Fig. 3. Signal-space diagram for the user2 QPSK 1st bit with user1 1st bit correct decoding $(00,10),(10,11)$.

For class I, Fig. 3 illustrates the decoding signal-space diagram for the user2 QPSK 1st bit $i_{3}$ after the corresponding user1 QPSK first bit $i_{1}$ has the correct decoding (e.g., $(00,10)$ and $(10,11)$ constellation points). For $(00,10)$, as decoding the user1 first bit $i_{1}$ correctly, it can be inferred from Fig. 2 that 
the noise is more than $(-d+d / \sqrt{A})$. Besides, when the noise goes beyond $d / \sqrt{A}$, a wrong decision will be made for bit $i_{3}$. Therefore, the decision boundary for decoding bit $i_{3}$ of $(00,10)$ in class I is $d / \sqrt{A}$. A bit error of $i_{3}$ will occur if the AWGN exceeds $d / \sqrt{A}$. This analytical result for bit $i_{3}$ can apply to $(01,10),(00,11),(01,11)$ cases.

For $(10,11)$, it can be seen from Fig. 2 that the AWGN should be less than $(d+d / \sqrt{A})$ in the condition of decoding the user1 first bit correctly. At the same time, the bit $i_{3}$ of $(10,11)$ will be detected erroneously, if the AWGN exceeds $d / \sqrt{A}$, as shown in Fig. 3. So the decision boundaries for bit $i_{3}$ of $(10,11)$ in class I are $d / \sqrt{A}$ and $(d+d / \sqrt{A})$. This analytical result for bit $i_{3}$ also applies to $(10,10),(11,10),(11,11)$ cases.

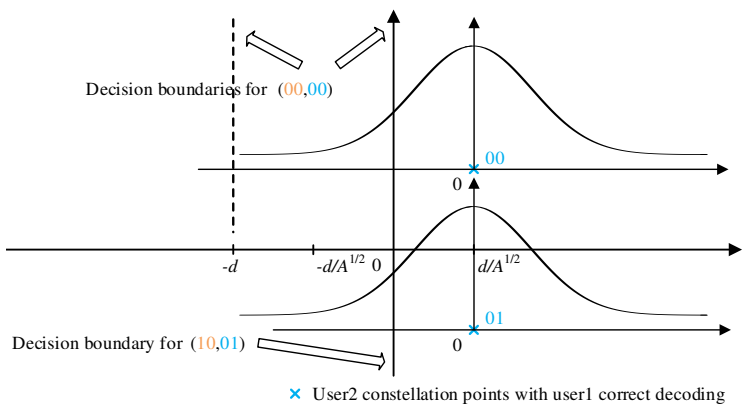

Fig. 4. Signal-space diagram for the user2 QPSK 1st bit with user1 1st bit correct decoding $(00,00),(10,01)$.

In a similar way, the decision boundaries of bit $i_{3}$ are $(-d-$ $d / \sqrt{A})$ and $-d / \sqrt{A}$ for $(00,00)$, as shown in Fig. 4. Meanwhile, $(01,00),(00,01)$ and $(01,01)$ cases have the same bit $i_{3}$ decision boundaries as $(00,00)$. For $(10,01)$ shown in Fig. 4 , the bit $i_{3}$ decision boundary is $-d / \sqrt{A}$, which is the same as $(10,00)$, $(11,00),(11,01)$ cases.

Consequently, the error probability of the bit $i_{3}$ in class I can be represented as follows

$$
\mathcal{P}_{i_{3} \mathrm{I}}=\frac{1}{4}\left[\operatorname{Pr}\left(\frac{d}{\sqrt{A}}<|n|\right)+\operatorname{Pr}\left(\frac{d}{\sqrt{A}}<|n|<d+\frac{d}{\sqrt{A}}\right)\right] .
$$

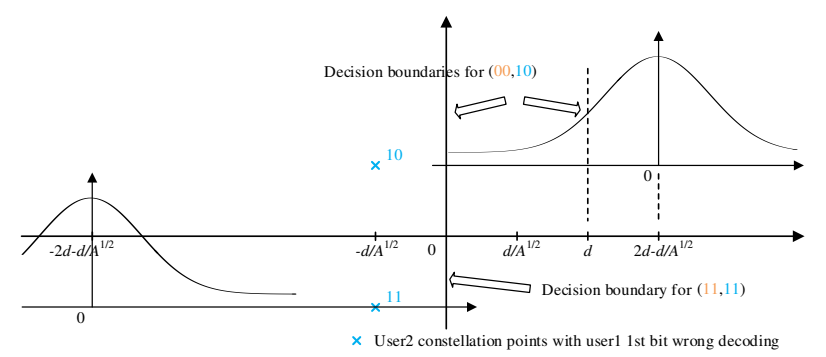

Fig. 5. Signal-space diagram for the user2 QPSK 1st bit with user1 1st bit wrong decoding $(00,10),(11,11)$.

For class II, Fig. 5 depicts the decoding signal-space diagram of $(00,10),(11,11)$ cases for the user2 QPSK 1st bit $i_{3}$, if decoding the corresponding user1 QPSK first bit $i_{1}$ wrongly. When decoding the bit $i_{1}$ erroneously, there will be error propagation which affects decoding the user2 first bit $i_{3}$. It is shown that the error propagation is $2 d$ for the $(00,10)$ case in Fig. 5, and the user2 constellation point (10) is transfered from $-d / \sqrt{A}$ to $(2 d-d / \sqrt{A})$ in the horizontal direction. If the bit $i_{1}$ is decoded erroneously in the $(00,10)$ case, the AWGN should be less than $(-d+d / \sqrt{A})$ as shown in Fig. 2. However, there will exist wrong decoding of bit $i_{3}$ for $(00,10)$, if the AWGN goes more than $(-2 d+d / \sqrt{A})$. So the decision boundaries for decoding the bit $i_{3}$ of $(00,10)$ in class II are $(-d+d / \sqrt{A})$ and $(-2 d+d / \sqrt{A})$. Through the same analysis, the $(01,10),(00,11)$, $(01,11)$ cases in class II have the same decision boundaries for decoding bit $i_{3}$.

For $(11,11)$ in Fig. 5 , as the wrong decoding of bit $i_{1}$, the user 2 constellation point (11) is transfered from $-d / \sqrt{A}$ to $(-2 d-d / \sqrt{A})$ in the horizontal direction, and the AWGN should be more than $(d+d / \sqrt{A})$ from Fig. 2. Thus, the error decision region for bit $i_{3}$ of $(11,11)$ is $(n>2 d+d / \sqrt{A})$, which is the same in $(10,10),(11,10),(10,11)$ cases.

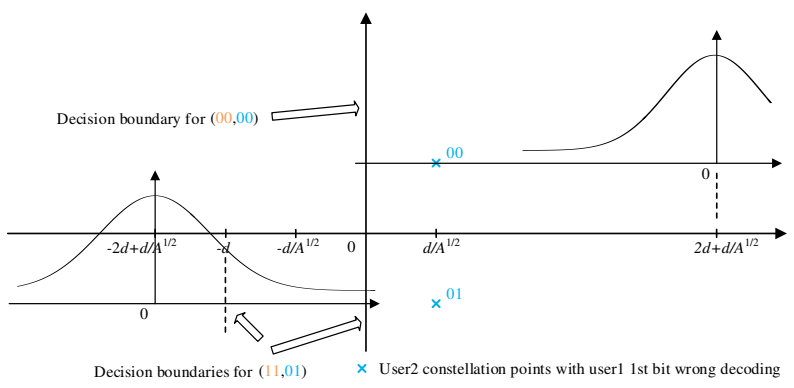

Fig. 6. Signal-space diagram for the user2 QPSK 1st bit with user1 1st bit wrong decoding $(00,00),(11,01)$.

In a similar way, in Fig. 6 the decision boundaries for bit $i_{3}$ of $(11,01)$ are $(d-d / \sqrt{A})$ and $(2 d-d / \sqrt{A})$, which can also apply to decoding the bit $i_{3}$ in $(10,00),(11,00),(10,01)$ cases. For $(00,00)$ shown in Fig. 6 , the bit $i_{3}$ decision boundary is $(-2 d-d / \sqrt{A})$, and $(01,00),(00,01),(01,01)$ cases have the same decision boundary.

Considering all these cases, the probability that the bit $i_{3}$ is in error in terms of class II can be expressed as follows

$$
\begin{aligned}
\mathcal{P}_{i_{3} \mathrm{II}}=\frac{1}{4} & {\left[\operatorname{Pr}\left(2 d+\frac{d}{\sqrt{A}}<|n|\right)\right.} \\
& \left.+\operatorname{Pr}\left(d-\frac{d}{\sqrt{A}}<|n|<2 d-\frac{d}{\sqrt{A}}\right)\right] .
\end{aligned}
$$

Finally, the exact average BER of user2 QPSK over an AWGN channel can be obtained from (4) and (5)

$$
\begin{array}{r}
\mathcal{P}_{2}=\frac{1}{2}\left[2 Q\left(\frac{d / \sqrt{A}}{\sqrt{P_{n} / 2}}\right)-Q\left(\frac{d+d / \sqrt{A}}{\sqrt{P_{n} / 2}}\right)+Q\left(\frac{2 d+d / \sqrt{A}}{\sqrt{P_{n} / 2}}\right)\right. \\
\left.+Q\left(\frac{d-d / \sqrt{A}}{\sqrt{P_{n} / 2}}\right)-Q\left(\frac{2 d-d / \sqrt{A}}{\sqrt{P_{n} / 2}}\right)\right] .
\end{array}
$$

\section{Simulation RESUltS AND ANALYSIS}

In order to verify the analytical results above, Monte Carlo simulations have been undertaken. In the Monte Carlo simulations, the two users apply the SC-FDM scheme at the transmitters, and they use the same time-frequency resources. Through AWGN channels, these two users' superposed signals are received by the BS. After decoding user1's signals, the SIC 


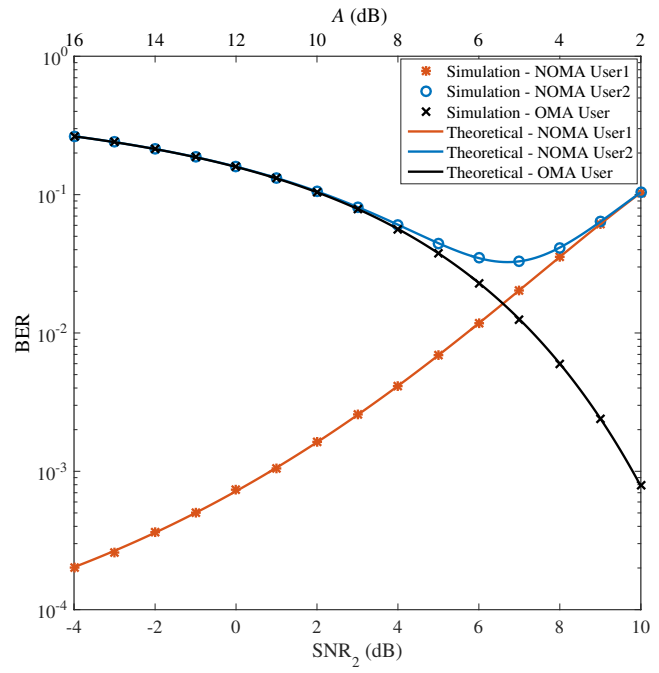

Fig. 7. BER performance comparisons in $\mathrm{SNR}_{1}=12 \mathrm{~dB}$.

process is implemented in the time domain before decoding user2's signals at the BS. The $\mathrm{SNR}_{1}$ is set as $12 \mathrm{~dB}$. As it is assumed that $P_{1}\left|h_{1}\right|^{2}>P_{2}\left|h_{2}\right|^{2}$ before, $\mathrm{SNR}_{2}$ is set in $[-4,10]$ $\mathrm{dB}$. The orthogonal multiple access (OMA) QPSK BER curve is also plotted as a comparison, i.e., one SC-FDMA user QPSK BER curve is included. The OMA user has the same SNR as $\mathrm{SNR}_{2}$. It should be noted that there is no IUI to the OMA user in our model, and the theoretical BER expression of the OMA user is $Q\left(\sqrt{\mathrm{SNR}_{2}}\right)$ [10].

Fig.7 shows the analytical and Monte Carlo simulated BER performances of the uplink QPSK NOMA system over an AWGN channel. It reveals perfect agreements between our exact closed-form expressions and Monte Carlo simulations. The bottom x-axis of Fig.7 represents the NOMA user2's and OMA user's SNR, and the top $\mathrm{x}$-axis represents the corresponding NOMA SNR difference $A(\mathrm{~dB})$. For example, when $\mathrm{SNR}_{2}=0 \mathrm{~dB}, A(\mathrm{~dB})$ is $12 \mathrm{~dB}$. Especially, if we suppose that $P_{1}=P_{2}, A$ denotes the channel difference, i.e., $A=\left|h_{1}\right|^{2} /\left|h_{2}\right|^{2}$. The simulation results indicate that the SNR difference of the two NOMA users has severe influence on users' BER performances. NOMA user1 BER increases markedly as $A$ decreases, i.e., $\mathrm{SNR}_{2}$ rises. This is because the IUI from user2 to user1 grows with the rise of $\mathrm{SNR}_{2}$. However, the user2 BER drops steadily to the rock bottom at $\mathrm{SNR}_{2}=6.71 \mathrm{~dB}(A=5.29 \mathrm{~dB})$ but gradually rises again with the decline of $A$. The intuitive explanation about the user2 BER curve tendency is that when $\mathrm{SNR}_{2}<6.71 \mathrm{~dB}(A>5.29$ $\mathrm{dB})$, the BER of user2 falls with the increase of $\mathrm{SNR}_{2}$. Meanwhile, the error propagation from user1 has no significant impact on the user2 BER performance because the user1 BER is on the small side. Nevertheless, after $\mathrm{SNR}_{2}>6.71$ $\mathrm{dB}(A<5.29 \mathrm{~dB})$, the error propagation from user1 is large enough to have a dominant influence on the user2 BER performance. Consequently, BER of user2 ascends again due to the user1's error propagation. Compared with the OMA user, NOMA user2 almost has the same BER as the OMA user when the error propagation from user1 is small $\left(\mathrm{SNR}_{2}<3\right.$ $\mathrm{dB})$. However, the BER performance gap between the NOMA user2 and the OMA user becomes larger, when the error propagation becomes stronger $\left(\mathrm{SNR}_{2}>3 \mathrm{~dB}\right)$.

\section{Conclusions}

NOMA has been recognized as a novel and promising technique for future radio access. This letter has presented and analyzed the closed-form BER expressions for QPSK in an uplink NOMA scheme over an AWGN channel. Corresponding simulation results validate the correctness of the derived expressions. As the QPSK BER expressions are formulated as a sum of $\mathrm{Q}$ functions, they can also be easily extended to the BER expressions in various fading cases with or without diversity reception.

\section{REFERENCES}

[1] S. M. R. Islam, N. Avazov, O. A. Dobre, and K. S. Kwak, "Power-Domain Non-Orthogonal Multiple Access (NOMA) in 5G Systems: Potentials and Challenges," IEEE Commun. Surveys Tuts., vol. PP, no. 99, pp. 1-1, 2016.

[2] Z. Ding, Y. Liu, J. Choi, Q. Sun, M. Elkashlan, C. L. I, and H. V. Poor, "Application of Non-Orthogonal Multiple Access in LTE and 5G Networks," IEEE Commun. Mag., vol. 55, no. 2, pp. 185-191, February 2017.

[3] Z. Yang, Z. Ding, P. Fan, and N. Al-Dhahir, "A general power allocation scheme to guarantee quality of service in downlink and uplink NOMA systems," IEEE Trans. Wireless Commun., vol. 15, no. 11, pp. 7244-7257, 2016.

[4] A. Li, A. Benjebbour, X. Chen, H. Jiang, and H. Kayama, "Uplink non-orthogonal multiple access (NOMA) with single-carrier frequency division multiple access (SCFDMA) for 5G systems," IEICE Trans. Commun., vol. 98, no. 8, pp. 1426-1435, 2015.

[5] Y. Endo, Y. Kishiyama, and K. Higuchi, "Uplink nonorthogonal access with MMSE-SIC in the presence of inter-cell interference," in International Symposium on Wireless Communication Systems (ISWCS), Paris, France, Aug. 2012, pp. 261-265.

[6] S. Islam and K. Kwak, "Outage capacity and source distortion analysis for NOMA users in 5G systems," Electron. Lett., vol. 52, no. 15, pp. 1344-1345, 2016.

[7] Z. Ding, P. Fan, and H. V. Poor, "Impact of User Pairing on 5G Nonorthogonal Multiple-Access Downlink Transmissions," IEEE Trans. Veh. Technol., vol. 65, no. 8, pp. 6010-6023, Aug 2016.

[8] H. Haci, H. Zhu, and J. Wang, "Performance of Nonorthogonal Multiple Access With a Novel Asynchronous Interference Cancellation Technique," IEEE Trans. Commun., vol. 65, no. 3, pp. 1319-1335, March 2017.

[9] 3GPP TR36.814 (V9.0.0), "Further advancements for EUTRA physical layer aspects," Mar. 2010.

[10] K. Cho and D. Yoon, "On the general BER expression of one- and two-dimensional amplitude modulations," IEEE Trans. Commun., vol. 50, no. 7, pp. 1074-1080, Jul 2002.

[11] W. T. Webb and L. Hanzo, Modern Quadrature Amplitude Modulation: Principles and applications for fixed and wireless channels: one. IEEE Press-John Wiley, 1994. 